\title{
Hybrid Macro/Microdiversity Techniques in the Reverse-Link Wireless Communication Networks
}

\author{
Abdulkareem Adinoyi and Halim Yanikomeroglu
}

\begin{abstract}
We investigate microdiversity-augmented macrodiversity techniques in wireless communication networks. The setup consists of $K$ widely separated access ports each carrying $N$ antennas. The conventional selection macrodiversity (Scheme I) and two proposed methods (Scheme II and Scheme III) for port selection are investigated and their performance are compared. Scheme II utilizes the advancement in distributed antennas and radio-on-fiber technologies whereas Scheme III utilizes these technologies in a way similar to the soft handover of CDMA systems. After the port selection, the microdiversity uses $n$ strongest signals $(n \leq N)$ of the selected port for diversity combining if practical constraints preclude the use of all $N$ signals.

Analytical performance expressions for the probability of error and outage probability for Scheme I are developed. Simulations are used to investigate Scheme II and Scheme III and it is observed that these latter schemes exhibit performance superiority over the conventional macrodiversity selection method. The power savings obtained using the proposed architecture and new selection schemes could be used either for coverage extension or to increase capacity in wireless networks.
\end{abstract}

Index Terms-Antenna architectures, hybrid macro/microdiversity, macrodiversity, microcellular, micro-diversity, Rayleigh, shadowing, wireless networks.

\section{INTRODUCTION}

$\mathbf{M}$ ICRODIVERSITY, through collocated antennas, has been used to combat small-scale fading. However, large-scale fading known as shadowing overwhelms a system with such closely spaced antennas. Emerging distributed and sectorized distributed antennas (SDA) [1] - [3] and the radioon-fiber ( $\mathrm{RoF})$ technologies [4], [5] are providing novel ways of handling signals received at widely separated antennas. These technologies are particularly suitable for microcellular systems and they could facilitate linking of distributed antennas or access ports to a central unit where signal-specific processing could then be performed. This paper proposes microdiversity-augmented macrodiversity architecture to combat small and large scale fading phenomena.

The proposed system works as follows. In the $K$-macro/ $N$ microdiversity-antenna structure, the macrodiversity mechanism could select the best among $K$ ports, using certain criteria, and of the $N$ signals available at that port, $n$ strongest are selected for diversity combining. Selecting a subset of spatial diversity signals to combine is known as generalized selection

Manuscript received December 20, 2004; revised November 18, 2005 and June 25, 2006; accepted July 31, 2006. The associate editor coordinating the review of this letter and approving it for publication was A. Yener. This work was presented in part at the IEEE Int'l. Conf. on Commun. (ICC'04) and the IEEE Wireless Commun. and Networking Conf. (WCNC'05).

The authors are with the Broadband Communications and Wireless Systems (BCWS) Centre, Department of Systems and Computer Engineering, Carleton University, Canada (email: \{adinoyi, halim\}@ sce.carleton.ca).

Digital Object Identifier 10.1109/TWC.2006.04803 combining (GSC) and it was first applied against small scale fading [6]. The GSC is employed as the receiver processor for the microdiversity component since it represents a reasonable system complexity and cost compromise compared to a full blown maximal ratio combining (MRC) [7] [8].

The conventional scheme [9] uses only the macroscopic component as the basis for port selection. This selection criterion has been adopted with the setup described above and the technique is referred to as Scheme $\mathrm{I}$ in this paper. Analytical expressions for error rate and outage performance have been derived for this scheme. The advancement in distributed antenna (DA) technology and radio-on-fiber, and the availability of a fast scanning receiver for the GSC [8], would allow for fast tracking of the composite fading. Therefore, this paper investigates the performance of a system that adopts these new technologies; we propose and evaluate the performance of Scheme II and Scheme III that consider port selection based on the composite fading as opposed to the selection mechanism in the conventional scheme [9].

The system architecture proposed in the context of microcellular networks helps avoid cell-splitting often used for improving the system performance in conventional cellular networks. The proposed architecture employs radio access points which are linked to a central unit (CU) using the DA and RoF technologies instead of deploying many expensive base stations. The two proposed architectures are referred to as Scheme II and Scheme III. Scheme II shows performance superiority over the conventional macro-selection method (Scheme I). Furthermore, through simulations the paper explores macroscopic MRC in microcellular systems referred to as Scheme III. Since this scheme processes signals across the macro-diversity ports it can be viewed as an extension of the CDMA cellular soft handover to non-CDMA systems.

\section{Description of System And Channel Models}

Fig. 1 shows the proposed antenna scheme in a wireless network area. In the SDA format a separate feeder exists between each branch (microdiversity antenna) and the CU. Hence, a total of $N \times K$ distinct signals are received at the $\mathrm{CU}$ that enables the separation of the received signals. All the signal-specific processing (such as demodulation) is performed at the $\mathrm{CU}$; this results in relatively inexpensive ports. The channel between a port and a user is modelled as Rayleigh flat fading plagued with lognormal shadowing.

Let the signal received at the $l$-th branch of the $k$-th port in the $K$-macro/ $N$-microdiversity-antenna architecture be denoted as $y_{k, l}$. Therefore, $y_{k, l}$ can be expressed as

$$
y_{k, l}=\sqrt{\gamma_{k}} \widetilde{\alpha}_{k, l} x+n_{k, l},
$$




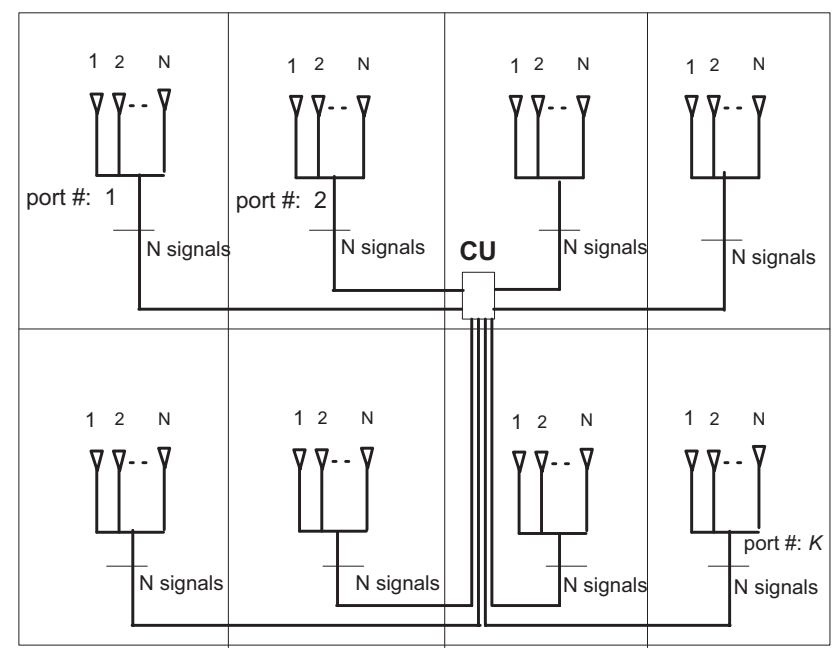

Fig. 1. Antenna layout for the hybrid macro/microdiversity schemes in a wireless network.

where $\mathbf{y}_{k}=\left\{y_{k, 1}, y_{k, 2}, \cdots, y_{k, N}\right\}$ is the set of received signals at port $k, x$ is the transmitted signal, and $\mathbf{n}_{k}=$ $\left\{n_{k, 1}, n_{k, 2}, \cdots, n_{k, N}\right\}$ is the set of the two-dimensional additive white Gaussian noise (AWGN) components. The branch gains $\sqrt{\gamma_{k}} \widetilde{\alpha}_{k, l}$ represented as $\alpha_{k, l}$, are the composite lognormal and Rayleigh distributed random variables with a common local mean $E\left[\alpha_{k, l}^{2}\right]=\Omega_{k}$. Therefore, the local mean received signal-to-noise ratio (SNR) per branch is $\frac{E_{s}}{N_{0}} \Omega_{k}$, where $E_{s}$ is the signal energy and $N_{0}$ is the single-sided power spectral density of the AWGN. The instantaneous SNR at each branch is $\Gamma_{k, l}=\alpha_{k, l}^{2} \frac{E_{s}}{N_{0}}$.

\section{MACROdiversity SELECTION SCHEMES}

The macrodiversity selection methods are presented in the following:

\section{A. Scheme I: The Conventional Macrodiversity}

The local mean SNR is the basis for selecting the port whose signals are processed.

- Obtain the local means $\Omega_{k}, 1 \leq k \leq K$, noting that shadowing is same for all branches in a port.

- Identify the port $k^{*}$ with the largest local mean; i.e., $k^{*}=$ $\underbrace{\operatorname{argmax}}_{1<k<K}\left\{\Omega_{k}\right\}$.

- Select $n \leq N$ branches of this port $k^{*}$ with the largest $\Gamma_{k^{*}, l}$.

However, based on microcellular architecture and distributed antenna systems, the following strategies can be adopted to further take advantage of all the distinct $N \times K$ signals that are collected at the $\mathrm{CU}$.

\section{B. Scheme II}

At each branch of a port, the SNR depends on both the macroscopic and microscopic fading; we can exploit this fact in making an informed port selection decision rather than deciding based only on the local mean SNR.
- Choose the port $k^{*}$ that has the largest overall aggregate SNR ( $N$-aggregate); i.e., $k^{*}=\underbrace{\operatorname{argmax}}_{1 \leq k \leq K}\left\{\sum_{l=1}^{N} \Gamma_{k, l}\right\}$.

- Select $n \stackrel{\leq}{\leq}$ branches of this port $k^{*}$ with the largest $\Gamma_{k^{*}, l}$.

In a suboptimal implementation of Scheme II, the port selection can be performed on the highest branch SNR;

- Identify $k^{*}=\underbrace{\operatorname{argmax}}_{1 \leq k \leq K}\{\underbrace{\max }_{1 \leq l \leq L} \Gamma_{k, l}\}$.

- Select $n \leq N$ branches of this port $k^{*}$ with the largest $\Gamma_{k^{*}, l}$.

A third realization of Scheme II which appears as the reasonable receiver option (selection based on $n$-aggregate) is the following:

- Arrange each port $N$ signals in descending order.

- Choose the port $k^{*}$ that has the largest sum of $n$ branch SNRs; i.e., $k^{*}=\underbrace{\operatorname{argmax}}_{1 \leq k \leq K}\left\{\sum_{l=1}^{n} \Gamma_{k, l}\right\}$.

However, in practice, implementation consideration may make the first and second algorithms more attractive.

\section{Scheme III}

This scheme, by the virtue of the $N \times K$ signals available at the $\mathrm{CU}$, selects signals across the ports to process.

- Arrange all the $N \times K$ signals in ascending order of their SNR $\left(\Gamma_{k, l}\right)$.

- Select the largest $n$ out of the $N \times K$ branches.

In all cases, MRC technique is used to process the $n$ selected branches, where $1 \leq n \leq N$.

\section{Error Performance Analysis for Scheme I}

Let $\beta, n, \gamma^{*}$ represent the sum of the combined Rayleigh fading branches at the selected port, the number of microdiversity branches selected, and the shadowing component at the selected port, respectively. Then $\beta=\sum_{i=1}^{n} \widetilde{\alpha}_{k^{*}, i}^{2}$ and $\gamma^{*}=10^{g^{*} / 10}$, where $g^{*}$ is the gaussian variable that forms the realization of the shadowing component for the selected port. The symbol error probability (SEP) for MPSK modulation conditioned on the fade samples can be expressed for GSC as

$$
P_{\operatorname{mpsk}}\left(e \mid \beta, g^{*}\right) \approx h \times \operatorname{erfc}\left(\sqrt{\beta \gamma^{*} \sin ^{2}\left(\frac{\pi}{M}\right) \frac{E_{s}}{N_{0}}}\right),
$$

where for BPSK, $h=1 / 2$ yields an equality, and for higher MPSK constellations, $h=1$.

In (2) the error performance is conditioned on the parameters $\beta$ and $g^{*}$. Therefore, (2) is averaged over the relevant two PDFs to obtain the unconditional error performance as 
follows:

$$
\begin{aligned}
P_{\mathrm{mpsk}}(e) & =\int_{0}^{\infty} P_{\mathrm{mpsk}}\left(e \mid \beta, g^{*}\right) \\
& \times \int_{-\infty}^{\infty} p(\beta ; n) p_{G^{*}}\left(g^{*} ; K\right) d g^{*} d \beta \\
& =\underbrace{\infty}_{-\infty} p_{G^{*}}\left(g^{*} ; K\right) \\
& \times \underbrace{\int_{0}^{\infty} p(\beta ; n) P_{\mathrm{mpsk}}\left(e \mid \beta, g^{*}\right) d \beta}_{P_{\mathrm{M}}\left(e \mid g^{*}\right)} d g^{*} .
\end{aligned}
$$

The PDF of $\beta$ can be expressed, by the help of the results in [7] as

$$
\begin{aligned}
p(\beta ; n) & =\left(\begin{array}{c}
N \\
n
\end{array}\right)\left[\frac{\beta^{n-1} e^{-\beta}}{(n-1) !}+\sum_{l=1}^{N-n} C_{l}^{N-n}(-1)^{n+l-1}\right. \\
& \left.\times\left(\frac{n}{l}\right)^{n-1} e^{-\beta}\left(e^{-\frac{l \beta}{n}}-\sum_{p=0}^{n-2} \frac{1}{p !}\left(-\frac{l \beta}{n}\right)^{p}\right)\right],(4)
\end{aligned}
$$

where $\left(\begin{array}{c}N \\ n\end{array}\right)=\frac{N !}{(N-n) ! n !}$. Using (4) in (3), the expression for the SEP of MPSK conditioned on the lognormal shadowing can be expressed as ${ }^{1}$

$$
\begin{aligned}
P_{\mathrm{M}}\left(e \mid g^{*}\right) & =h\left(\begin{array}{c}
N \\
n
\end{array}\right)\left[\frac{1}{2^{2 n-2}} \sum_{j=1}^{n} C_{n-1}^{2 n-j-1}\left(\frac{2}{1+\sqrt{\delta_{1}}}\right)^{j}\right. \\
& \times \frac{1}{\left(1+\gamma^{*} \lambda\right)^{n}}+\sum_{l=1}^{N-n} \frac{2 \eta(l, n, N)}{\left(l / n+1+\gamma^{*} \lambda\right)\left(1+\sqrt{\delta_{2}}\right)} \\
& -\sum_{l=1}^{N-n} \eta(l, n, N) \sum_{p=0}^{n-2} \frac{4(-l / n)^{p}}{\left[4\left(1+\gamma^{*} \lambda\right)\right]^{p+1}} \\
& \left.\times \sum_{q=1}^{p+1} C_{p}^{2(p+1)-q-1}\left(\frac{2}{1+\sqrt{\delta_{1}}}\right)^{q}\right]
\end{aligned}
$$

where, $\eta(l, n, N)=\left(\begin{array}{c}N-n \\ l\end{array}\right)(-1)^{n+l-1}(n / l)^{n-1}, \delta_{1}=$ $\frac{\gamma^{*} \lambda}{1+\gamma^{*} \lambda}, \delta_{2}=\frac{\gamma^{*} \lambda}{1+\gamma^{*} \lambda+l / n}$, and $\lambda=\sin ^{2}\left(\frac{\pi}{M}\right) \frac{E_{s}}{N_{0}}$.

For Scheme I we define $G^{*}=\max \left\{G_{1}, G_{2}, \cdots, G_{K}\right\}$ as the local mean value which corresponds to the port selected for the macroscopic diversity. Each $G_{k}$ has a Gaussian distribution with a certain area mean $\mu_{k} \mathrm{~dB}$ and standard deviation $\sigma_{k} \mathrm{~dB}$. For identically and independently distribution, the probability density function (PDF) of $G^{*}$ can be written [9] as

$$
\begin{aligned}
p_{G^{*}}\left(g^{*} ; K\right) & =\frac{K}{\sqrt{2 \pi \sigma^{2}}} \exp \left(-\frac{\left(g^{*}-\mu\right)^{2}}{2 \sigma^{2}}\right) \\
& \times\left[1-\frac{1}{2} \operatorname{erfc}\left(\frac{g^{*}-\mu}{\sqrt{2 \sigma^{2}}}\right)\right]^{K-1},
\end{aligned}
$$

where a simplifying assumption of $\mu_{k}=\mu$, for all $k$, is made.

Finally, the error probability can be obtained by evaluating the integral in (3), that is,

$$
P_{\mathrm{mpsk}}(e) \approx \int_{-\infty}^{\infty} P_{\mathrm{M}}\left(e \mid g^{*}\right) p_{G^{*}}\left(g^{*} ; K\right) d g^{*} .
$$

\footnotetext{
${ }^{1}$ See Appendix I for the derivation of $P_{\mathrm{mpsk}}\left(e \mid g^{*}\right)$.
}

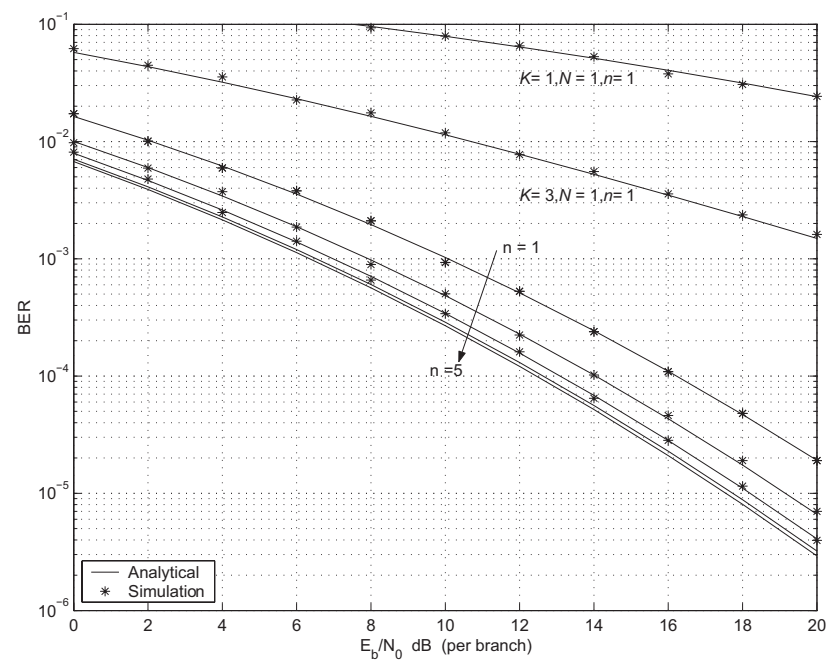

Fig. 2. Performance of BPSK in selection macrodiversity and GSC-based microdiversity (Scheme I) with lognormal shadowing $(\sigma=12 \mathrm{~dB})$ and Rayleigh fading; $K=3, N=5,1 \leq n \leq N$.

The integral in (7) can be evaluated numerically using standard mathematical softwares such as Mathematica or Matlab, evaluating the integral in a closed form is an open question.

\section{A. Numerical Results}

Numerical examples are provided in Fig. 2 for Scheme I. Analytical and simulation (denoted as $(*)$ ) results are shown for the bit error probability (BER) versus $E_{b} / N_{0}$ per branch. The BPSK modulation method is employed. The antenna configuration is $[K=3, N=5,1 \leq n \leq N]$. The scenarios where no microdiversity $[K=3, N=1, n=1]$ is employed and where neither macrodiversity nor microdiversity [ $K=1, N=1, n=1]$ is used are also shown for comparison purposes. The value of $\sigma$ is $12 \mathrm{~dB}$. Other values for $\sigma$ and different network configurations are considered but not shown due to space constraints. In all cases, the results obtained from the simulation campaigns agree well with the analytical ones.

The performance superiority of the hybrid schemes over these two reference scenarios is observed (Fig. 2); the gain of the hybrid schemes over the channel employing no form diversity is significant. It is observed that a fixed gain in SNR of about $2 \mathrm{~dB}$ is obtained for the system when $n$ is increased from one to two, for all $K$ and $N$ values considered. A gain of $1 \mathrm{~dB}$ is generally observed when $n$ is increased from 2 to 3. The same trend is observed for the other $K$ and $N$ combinations that are examined.

In addition to the presented results, higher constellation sizes (e.g., 8-PSK and 16-PSK) and different antenna configurations have exhibited the same trend of agreement between the simulations and analysis (therefore, they are not shown here for brevity of presentation).

Simulations are used to evaluate the performance of the algorithms described as Scheme II ( $N$-aggregate) and Scheme III. The performance of the two algorithms is compared with the simulated results for conventional Scheme I. In Fig. 3, three sets of curves are shown for the following network parameters $[K=2, N=5, n=1],[K=2, N=5, n=3]$, 


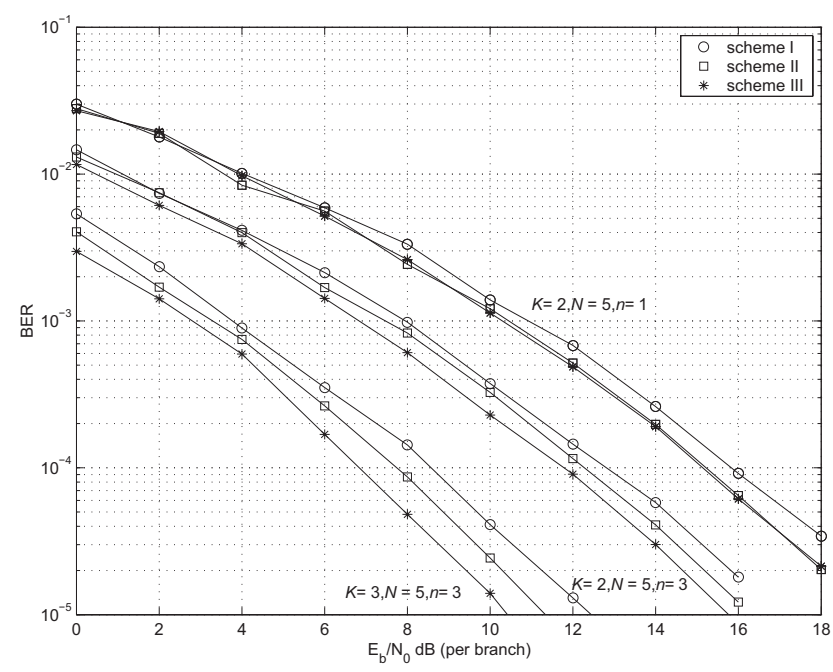

Fig. 3. Performance comparison of the hybrid macro/microdiversity schemes with $\sigma=8 \mathrm{~dB}$ for different configurations for BPSK.

and $[K=3, N=5, n=3]$. The shadowing standard deviation $\sigma$ is set to $8 \mathrm{~dB}$. It is observed that for the network $[K=3, N=5, n=3]$, Scheme III has about $1.5 \mathrm{~dB}$ advantage over Scheme $\mathrm{I}$ at $\mathrm{BER}=10^{-4}$. Considering the case $[K=2, N=5, n=3$ ], Schemes II and III perform better than Scheme I, but the gain margins are reduced. At lower error rates, the proposed schemes yield more gains over the conventional scheme. For instance, for Scheme III the gain is close to about $2 \mathrm{~dB}$ at $\mathrm{BER}=10^{-5}$ for $[K=3, N=5, n=$ $3]$.

The performance of Scheme III represents a lower bound for the hybrid schemes since in Scheme III the best $n$ branches is always selected which is not the case for Scheme II which explains the superior performance of Scheme III over Scheme II. On the other hand, the performance improvement of Scheme II over Scheme I comes from the way the received signals are treated without the need for extra ordinary signal processing, demonstrating that with modest extra processing efforts, an additional gain as high as $2 \mathrm{~dB}$ can be obtained in the proposed hybrid macro/microdiversity schemes. The performance of Scheme II based on $N$-aggregate SNR is compared with that based on best $n$-aggregate SNR for different network configurations and $\sigma$ values (Figs. 4 and 5 ). It is observed that the two modes of selecting port have comparable performance for the network configurations and parameters considered. Therefore, it is reasonable to compare the performance of $N$ aggregate of Scheme II with that of Scheme III as it is done in Fig. 3.

\section{Outage Probability Calculation}

Expression for calculating the quasi-static outage probability for a user in the microdiversity augmented macrodiversity architecture (Scheme I) is presented in this section. An outage is said to occur when a certain link quality metric (for example, bit error rate of a user) becomes worse than a required threshold value. The instantaneous SNR can be expressed as $\beta \gamma^{*} \frac{E_{b}}{N_{0}}$ and hence, the corresponding conditional bit error rate

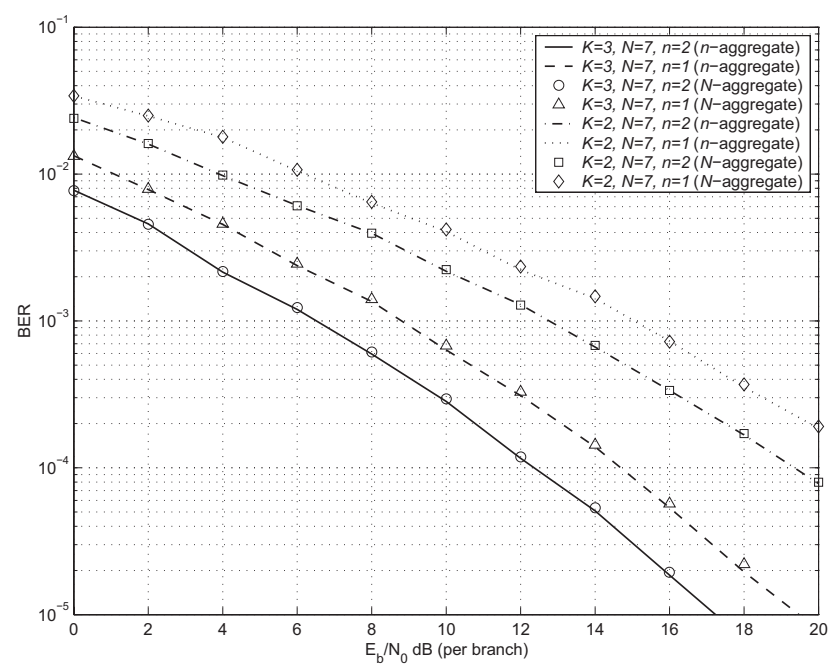

Fig. 4. Performance comparison of port selection based on $N$-aggregate and $n$-aggregate for different network configurations and for $\sigma=12 \mathrm{~dB}$.

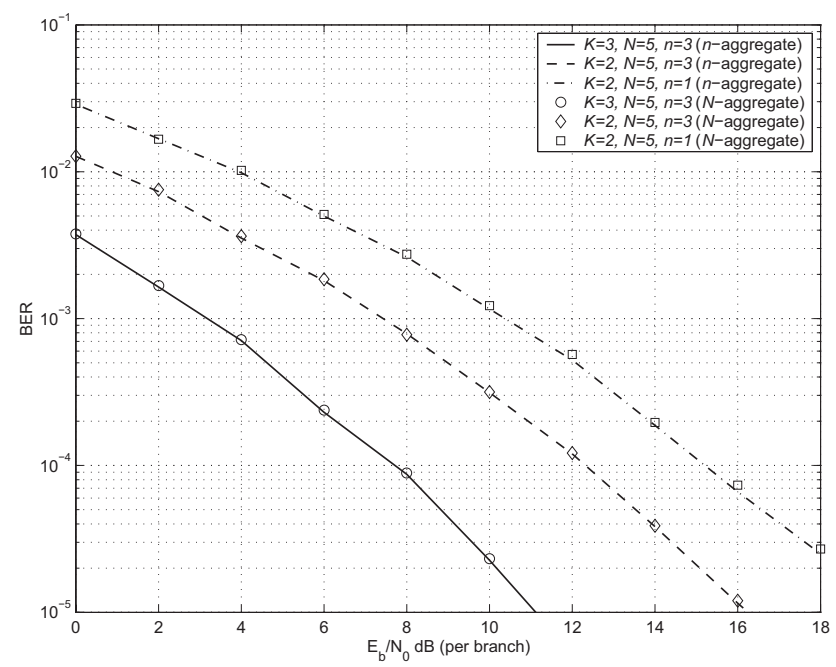

Fig. 5. Performance comparison of port selection based on $N$-aggregate and $n$-aggregate for different network configurations and for $\sigma=8 \mathrm{~dB}$.

for $\mathrm{QPSK}^{2}, P_{\mathrm{qpsk}}\left(e \mid \beta, g^{*}\right)$ is

$$
P_{\mathrm{qpsk}}\left(e \mid \beta, g^{*}\right)=\frac{1}{2} \operatorname{erfc}\left(\sqrt{\beta \gamma^{*} E_{b} / N_{0}}\right) \text {. }
$$

Note that the probability of $P_{\mathrm{qpsk}}\left(e \mid \beta, g^{*}\right)$ being greater than a set threshold, say $\zeta$, is equivalent to the probability of having $\beta$ that is less than a threshold $\beta_{t h r}$ (corresponding to $\zeta$ ) for a given $g^{*}$. This threshold value of $\beta$ can be expressed as $\beta_{t h r}=\left[\operatorname{erfc}^{-1}(2 \zeta)\right]^{2} / \gamma^{*} \frac{E_{b}}{N_{0}}$.

With this representation, the quasi-static outage probability can be obtained by averaging over the relevant PDFs

$$
\begin{aligned}
P_{\text {outage }} & =\int_{0}^{\beta_{t h r}}\left[\int_{-\infty}^{\infty} p(\beta ; n) p_{G^{*}}\left(g^{*} ; K\right) d g^{*}\right] d \beta \\
& =\int_{-\infty}^{\infty} p_{G^{*}}\left(g^{*} ; K\right) \underbrace{\int_{0}^{\beta_{t h r}} p(\beta ; n) d \beta}_{I_{1}} d g^{*}
\end{aligned}
$$

\footnotetext{
${ }^{2}$ Note that the BER of QPSK is equal to that of BPSK.
} 


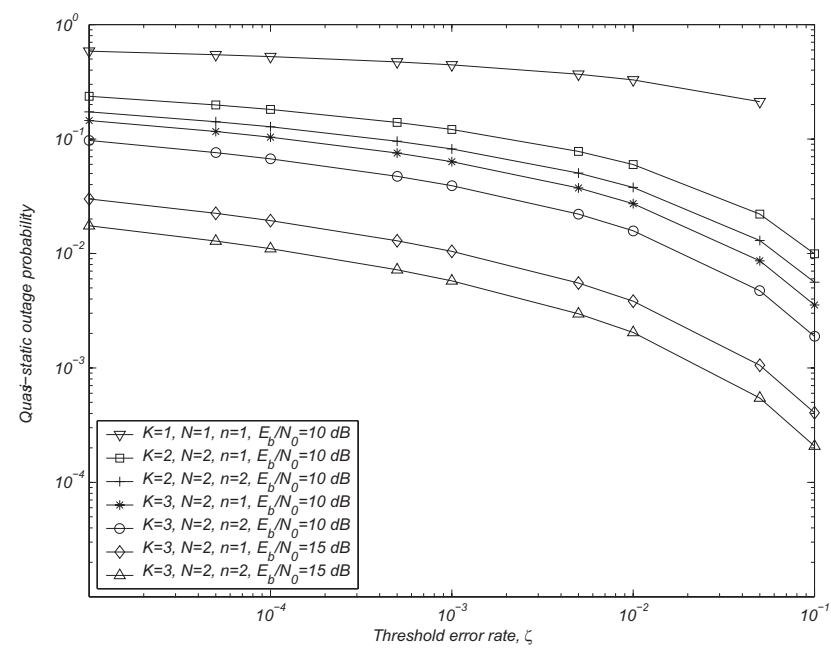

Fig. 6. Outage probability for QPSK modulation when Scheme I is employed $(\sigma=6 \mathrm{~dB})$.

The integral denoted as $I_{1}$ can be expressed in the following form using (4) as

$$
\begin{aligned}
I_{1} & =\left(\begin{array}{c}
N \\
n
\end{array}\right)\left[1-e^{-\beta_{t h r}} \sum_{k=0}^{n-1} \frac{\beta_{t h r}^{k}}{k !}+\sum_{l=1}^{N-n} \eta(l, n, N)\right. \\
& \times\left(\frac{1-e^{-\beta_{t h r}\left(\frac{l+n}{n}\right)}}{\frac{n+l}{n}}-\sum_{p=0}^{n-2}\left(\frac{-l}{n}\right)^{p}\right. \\
& \left.\left.\times\left(1-e^{-\beta_{t h r}} \sum_{t=0}^{p} \frac{\beta_{t h r}^{t}}{t !}\right)\right)\right] .
\end{aligned}
$$

Equations (6), (9) and (10) can be used to numerically evaluate the average outage probability. Outage for other modulation schemes can similarly be obtained.

The curves in Fig. 6 show the outage probability versus threshold bit error rate of QPSK for different values network configurations $[K, N$, and $n]$ and different values of $E_{b} / N_{0}$. We note that to attain low error rates we require very large $E_{b} / N_{0}$ in channels subjected to fading and shadowing, but the combination of microdiversity and macrodiversity can be used to reduce this power requirements. We also observe that for a given error rate threshold the amount of reduction in outage increases with the number of diversity branches. It is observed that there is a significant reduction in outage with $[K=2, N=2, n=1]$ over the case $[K=1, N=1, n=1]$, although, at each processing instant, one $\mathrm{RF}$ detection resource is employed.

\section{CONCLUSION}

The reverse-link performance of wireless communication systems employing a hybrid microdiversity and macrodiversity structure has been studied, where $K$ access ports, each carrying $N$ antennas, are deployed in a coverage region of a central unit (CU).

The paper provides derivation of error rate and outage expressions for the hybrid diversity scheme realized within the conventional macrodiversity framework. Simulations are used to validate the derived expressions. In addition, the feasibility of SDA and RoF techniques where the signals received at ports are transported through a broadband backbone to a $\mathrm{CU}$ is assumed. This way the CU has $K \times N$ separable signals for each user. New port and branch selection strategies for the hybrid macro/microdiversity structure are investigated and they exhibit superior performance over the conventional macroselection method. The hybrid macro/microdiversity architecture with the proposed port and branch selection schemes result in power savings which could help in cost-effective coverage extension or capacity boosting in wireless networks.

\section{ACKNOWLEDGMENT}

The authors would like to thank Prof. Sergey Loyka, School of Information Technology and Engineering, University of Ottawa, Canada, for his valuable input during the early stage of this work.

\section{APPENDIX}

The symbol error probability (SEP) for MPSK modulation conditioned on the fade samples can be expressed for GSC as

$$
P_{\text {mpsk }}\left(e \mid \beta, g^{*}\right) \approx h \times \operatorname{erfc}\left(\sqrt{\beta \gamma^{*} \lambda}\right) .
$$

The expression (4) is treated as $p(\beta, n)=p_{1}(\beta, n)+$ $p_{2}(\beta, n)+p_{3}(\beta, n)$ where,

$$
\begin{aligned}
& p_{1}(\beta, n)=\left(\begin{array}{c}
N \\
n
\end{array}\right) \frac{\beta^{n-1} e^{-\beta}}{(n-1) !} \\
& p_{2}(\beta, n)=\left(\begin{array}{c}
N \\
n
\end{array}\right) \sum_{l=1}^{N-n} \eta(l, n, N) e^{-\beta(1+l / n)}, \\
& p_{3}(\beta, n)=\left(\begin{array}{c}
N \\
n
\end{array}\right) \sum_{l=1}^{N-n} \eta(l, n, N) \sum_{p=0}^{n-2} \frac{1}{p !}\left(\frac{-l \beta}{n}\right)^{p} e^{-\beta} .
\end{aligned}
$$

Then $P_{\mathrm{M}}\left(e \mid g^{*}\right)=P_{1}+P_{2}+P_{3}$. Evaluation of the integral in $P_{\mathrm{mpsk}}\left(e \mid g^{*}\right)=\int_{0}^{\infty} P_{\mathrm{mpsk}}\left(e \mid \beta, g^{*}\right) p(\beta, n) d \beta$ is performed as

$$
P_{1}=h\left(\begin{array}{c}
N \\
n
\end{array}\right) \int_{0}^{\infty} \frac{\beta^{n-1} e^{-\beta}}{(n-1) !} \operatorname{erfc}\left(\sqrt{\beta \gamma^{*} \lambda}\right) d \beta .
$$

Let $\delta_{1}=\frac{\gamma^{*} \lambda}{1+\gamma^{*} \lambda}$ and $y=\beta\left(1+\gamma^{*} \lambda\right)$, then $y \delta_{1}=\beta \gamma^{*} \lambda$. With this representation (13) can be expressed as

$$
P_{1}=h\left(\begin{array}{c}
N \\
n
\end{array}\right) \int_{0}^{\infty} \frac{y^{n-1} e^{-y \delta_{1} /\left(\gamma^{*} \lambda\right)}}{(n-1) !\left(1+\gamma^{*} \lambda\right)^{n}} \operatorname{erfc}\left(\sqrt{y \delta_{1}}\right) d y .
$$

It can be shown that $\frac{\delta_{1}}{\gamma^{*} \lambda}=\left(1-\delta_{1}\right)$ and, afterwards, (14) is expressed in the following form [10]:

$$
\begin{array}{r}
\frac{1}{2(W-1) !} \int_{0}^{\infty} \operatorname{erfc}(\sqrt{x y}) e^{-y(1-x)} y^{W-1} d y \\
=\frac{1}{2^{2 W}} \sum_{j=1}^{W} C_{W-1}^{2 W-j-1}\left(\frac{2}{1+\sqrt{x}}\right)^{j} .
\end{array}
$$

From here $P_{1}$ is obtained in a straight forward manner which is the first part of (5).

$$
P_{2}=\int_{0}^{\infty} \operatorname{erfc}\left(\sqrt{\beta \gamma^{*} \lambda}\right)\left(\begin{array}{c}
N \\
n
\end{array}\right) \sum_{l=1}^{N-n} \eta(l, n, N) e^{-\beta(1+l / n)} d \beta .
$$


Let $\delta_{2}=\frac{\gamma^{*} \lambda}{\left(l / n+1+\gamma^{*} \lambda\right)}$ and $\Gamma=\beta\left(l / n+1+\gamma^{*} \lambda\right)$ then (16) can be written in the following form

$$
\begin{aligned}
P_{2} & =\left(\begin{array}{c}
N \\
n
\end{array}\right) \sum_{l=1}^{N-n} \eta(l, n, N) \frac{1}{\left(l / n+1+\gamma^{*} \lambda\right)} \\
& \times \int_{0}^{\infty} \operatorname{erfc}\left(\sqrt{\delta_{2} \Gamma}\right) e^{-\Gamma \frac{(1+l / n)}{1+l / n+\gamma^{*} \lambda}} d \Gamma .
\end{aligned}
$$

It can be shown that $\frac{1+l / n}{1+l / n+\gamma^{*} \lambda}=1-\delta_{2}$, therefore,

$$
\begin{aligned}
P_{2} & =\left(\begin{array}{c}
N \\
n
\end{array}\right) \sum_{l=1}^{N-n} \eta(l, n, N) \frac{1}{\left(l / n+1+\gamma^{*} \lambda\right)} \\
& \times \int_{0}^{\infty} \operatorname{erfc}\left(\sqrt{\delta_{2} \Gamma}\right) e^{-\Gamma\left(1-\delta_{2}\right)} d \Gamma
\end{aligned}
$$

and hence,

$$
P_{2}=\left(\begin{array}{c}
N \\
n
\end{array}\right) \sum_{l=1}^{N-n} \eta(l, n, N) \frac{1}{\left(l / n+1+\gamma^{*} \lambda\right)} \frac{1}{\left(1+\sqrt{\delta_{2}}\right)}
$$

and finally, by doing similar manipulations as performed above, $P_{3}$ can be expressed as

$$
\begin{aligned}
P_{3} & =\int_{0}^{\infty} \operatorname{erfc}\left(\sqrt{\delta_{1} \omega}\right)\left(\begin{array}{c}
N \\
n
\end{array}\right) \sum_{l=1}^{N-n} \eta(l, n, N) \\
& \times \sum_{p=0}^{n-2}(-l / n)^{p} \frac{1}{\left(1+\gamma^{*} \lambda\right)^{p+1}} \frac{\omega^{p}}{p !} e^{-\omega\left(1-\delta_{1}\right)} d \omega \cdot(20)
\end{aligned}
$$

By interchanging the integral and the summation, the integration can be simplified to

$$
\begin{aligned}
P_{3} & =\left(\begin{array}{c}
N \\
n
\end{array}\right) \sum_{l=1}^{N-n} \eta(l, n, N) \sum_{p=0}^{n-2}(-l / n)^{p} \frac{1}{\left(1+\gamma^{*} \lambda\right)^{p+1}} \frac{1}{2^{2 p+1}} \\
& \times \sum_{j=1}^{p+1}\left(\begin{array}{c}
2(p+1)-j-1 \\
p
\end{array}\right)\left(\frac{2}{1+\sqrt{\delta_{1}}}\right)^{j} .
\end{aligned}
$$

Combining $P_{1}, P_{2}$, and $P_{3}$ gives the expression for $P_{\mathrm{M}}\left(e \mid g^{*}\right)$.

\section{REFERENCES}

[1] A. Salmasi and K. Gilhousen, "On the system design aspects of code division multiple access (CDMA) applied to digital cellular and personal communication networks," in Proc. IEEE Veh. Technol. Conf., May 1991, pp. 57-62.

[2] H. Yanikomeroglu and E. S. Sousa, "CDMA distributed antenna system for indoor wireless communications," in Proc. 2nd Int'l. Conf. on Universal Personal Commun., Oct. 1993, pp. 990-994.

[3] H. Yanikomeroglu and E. S. Sousa, "CDMA sectorized distributed antenna system," in Proc. IEEE Int'l. Symposium on Spread Spectrum Techniques and Applications, Sep. 1998, pp. 792-797.

[4] J.-S. Wu, J. Wu, and H.-W. Tsao, "A radio-over-fiber network for microcellular system application," IEEE Trans. Veh. Technol., vol. 47, no. 1, pp. 84-94, Feb. 1998.

[5] R. Ohmoto, H. Ohtsuka, and H. Ichikawa, "Fiber-optic microcell radio system with a spectrum delivery scheme," IEEE J. Select. Areas Commun., vol. 11, no. 7, pp. 1108-1117, Sep. 1993.

[6] N. Kong and L. B. Milstein, "Combined average SNR of a generalized diversity selection combining scheme," in Proc. IEEE Int'l. Conf. Commun., June 1998, pp. 1556-1560.

[7] M.-S. Alouini and M. K. Simon, "An MGF-based performance analysis of generalized selection combining over Rayleigh fading channels," IEEE Trans. Commun., vol. 48, no. 3, pp. 401-415, Mar. 2000.

[8] M. Z. Win and J. H. Winters, "Virtual branch analysis of symbol error probability for hybrid selection/maximal-ratio combining in Rayleigh fading," IEEE Trans. Commun., vol. 11, no. 49, pp. 1926-1934, Nov. 2001.

[9] A. A. Abu-Dayya and N. C. Beaulieu, "Micro- and macrodiversity MDPSK on shadowed frequency-selective channels," IEEE Trans. Commun., vol. 43, no. 8, pp. 2334-2343, Aug. 1995.

[10] S. H. Jamali and T. Le-Ngoc, Coded-Modulation Techniques for Fading Channels. Upper Sadddle River, NJ: Kluwer Academic Publishers, 1994. 